\title{
Concept Maps and Learning Objects
}

\author{
Leonel Iriarte Navarro ${ }^{1}$, Manuel Marco Such ${ }^{2}$, Daniel Morón Martín ${ }^{2}$, \\ Carlos Pérez-Sancho ${ }^{2}$, Pedro Pernías $\mathrm{Peco}^{2}$ \\ ${ }^{1}$ Department of Informatics, Agrarian University of Havana, Cuba \\ lineinfomed.sld.cu \\ ${ }^{2}$ Department of Languages and Informatics Systems, University of Alicante, Spain \\ mmarco@renr.es, Dani.Moron@ua.es, \\ cperez@dlsi.ua.es, p.pernias@universia.net
}

\begin{abstract}
Concept Maps constitute one of the tools mostly used in learning management due to the possibility that they offer to contextualize learning, share knowledge and for learning to learn. At the same time, many initiatives or standards are being developed at full speed, which make the contents in different platforms and learning environments compatible. In this paper, the need to combine the technique of concept maps with the initiatives for packing the contents developed by the IMS to produce more portable and powerful content is stated. A model to create tools for learning management is proposed.
\end{abstract}




\title{
Diagramas Conceptuales y Objetos de Aprendizaje
}

\author{
Leonel Iriarte Navarro ${ }^{1}$, Manuel Marco $\mathrm{Such}^{2}$, Daniel Morón Martín ${ }^{2}$, \\ Carlos Pérez-Sancho ${ }^{2}$, Pedro Pernías Peco $^{2}$ \\ ${ }^{1}$ Departmento de Informática, Universidad Agraria de la Habana, Cuba \\ lin@infomed.sld.cu \\ ${ }^{2}$ Departmento de Lenguajes y Sistemas Informáticos, Universidad de Alicante, España \\ mmarcoerenr.es, Dani.Moron@ua.es, \\ cperez@dlsi.ua.es, p.pernias@universia.net
}

\begin{abstract}
Resumen. Los Mapas conceptuales constituyen una de las herramientas más utilizadas en la gestión del aprendizaje por la posibilidad que estos ofrecen de contextualizar el aprendizaje, compartir conocimiento, y para aprender a aprender. Al mismo tiempo se desarrollan a gran velocidad múltiples iniciativas o estándares que permiten compatibilizar los contenidos desarrollados en diferentes plataformas y entornos educativos. En este trabajo se destaca la necesidad de combinar las técnicas de diagramas conceptuales con los iniciativas para el empaquetamiento de contenidos desarrollada por IMS con el fin de producir contenidos mas portables y potentes. Se propone un modelo sirve de base para la creación de herramientas destinadas a la gestión del aprendizaje.
\end{abstract}

\section{Introducción}

Con la explosión de las nuevas tecnologías y el surgimiento de potentes métodos y herramientas para gestionar el conocimiento, se generan cada día mas comunidades virtuales que superan los límites de la distancia y ponen en función de profesores y estudiantes, la mayoría de los servicios del proceso docente tradicional. A pesar de los éxitos alcanzados, el proceso de aprendizaje se ve afectado porque muchos profesores e instituciones intentan aplicar en las nuevas circunstancias, las mismas estructuras de aprendizaje desarrolladas por ellos para la educación tradicional. Para generar contenidos acorde al desarrollo actual es necesario cambiar nuestro modelo mental e incorporar la idea de que es necesario ubicar al estudiante como responsable de su autonomía intelectual y de la gestión del conocimiento que el necesite [1] . Es muy importante que el contenido que se genere sea adaptable a las características de cada estudiante y al mismo tiempo que el profesor sea capaz de coordinar todas las acciones de la comunidad virtual para ampliar las fronteras del conocimiento propiciando la compartición de experiencias.

Durante la década de los setenta, surge en la universidad de Cornell, Estados Unidos, la teoría de los mapas conceptuales como una respuesta a la teoría del aprendizaje desarrollada por Ausubel, en lo referente a la evolución de las ideas previas que poseen los estudiantes para lograr nuevo conocimiento. Estas técnicas han constituido desde entonces una herramienta de gran utilidad para profesores, investigadores de 
temas educativos, psicólogos, sociólogos, estudiantes y otras áreas donde se necesita tratar grandes volúmenes de información [2]. Los mapas conceptuales constituyen un resumen esquemático de lo que se ha aprendido, ordenado en forma jerárquica donde el conocimiento está organizado y representado en todos los niveles de abstracción. Esta teoría ha tomado gran auge en los últimos años donde se han desarrollado muchas herramientas informáticas que permiten su implementación de forma muy sencilla.

El diseño y construcción de los mapas conceptuales ha sido resuelto mediante estos sistemas automatizados que están disponles a los usuarios en las mas variadas formas, cada uno tiene sus formatos, su propia estructura y estilos. La mayoría de ellos produce salidas en formato HTML para permitir la navegación por los diferentes nodos del mapa.

La generalización de estas herramientas ha provocado que existan gran cantidad de conocimiento útil que está escondido en la Web que le llaman invisible, debido a que cada profesor o institución va produciendo sus propios mapas y estos no son publicados ni organizados de la forma más adecuada, de modo que puedan ser reutilizados en otro momento.

Muchas veces un concepto de un mapa se enlaza a un sitio Web donde el alumno no logra encontrar la información que necesita provocando que este pierda su esencia. Esto se produce porque en el momento del diseño el profesor no cuenta con alternativas que le permitan buscar información precisa y orientada al aprendizaje sobre un concepto.

Las herramientas existentes carecen de facilidades para implantar estructuras de representación del conocimiento de alta complejidad en los que la información pueda generarse de forma dinámica y este presente el aprendizaje cooperativo, estas se limitan solo a representar secuencias o jerarquías dada la propia naturaleza de los diagramas conceptuales.

Muchos de los problemas de compatibilidad y estructuración del aprendizaje que hemos planteado han encontrado una vía de solución en la creación de estándares que permiten la documentación, búsqueda y distribución de los contenidos educativos que se generan en los diferentes entornos [3]. Entre los estándares más importantes se encuentra el IMS desarrollado por el Global Learning Consortium [4] y a partir de este, el SCORM desarrollado por Advanced Distributed Learning Initiative [5] y el Institute of Electrical and Electronics Engineers [6].

Los estándares se basan en la definición de Objeto de aprendizaje como cualquier objeto digital que pueda ser reusado [7]. Para lograr la reusabilidad y la interoperabilidad de los materiales docentes, se han creado diferentes especificaciones que proporcionan esquemas para clasificar y empaquetar contenidos.

Existen cientos de repositorios de Objetos de Aprendizaje donde profesores y estudiantes encuentran mucho más fácil los contenidos y los pueden incluir en sus plataformas de desarrollo. Nosotros hemos creado un repositorio de objetos de aprendizaje para la enseñanza del idioma Español a partir de cursos a distancia elaborados por el Instituto Cervantes de España [8], mediante una plataforma llamada Aula Virtual de Español, desarrollada en la Universidad de Alicante, España. [9]. Esta posee mas de 2000 actividades docentes que los profesores y expertos podrán reutilizar en el diseño de nuevos cursos. [10] 
Han surgido varias especificaciones que definen estructuras estándares para intercambiar contenidos y estructuras de aprendizaje, lo que permite convertir estas en objetos de aprendizaje. Las más conocidas son las que ha desarrollado IMS [4]. La primera y más difundida es IMS Content Packaging (IMS-CP) [11], que permite organizar estructuras de contenidos básicamente jerárquicas. Luego se propuso IMS Simple Sequencing Specification (IMS-SS) [12] que esta basada en IMS-CP y permite definir secuencias de actividades docentes y las condiciones en que cada actividad debe ejecutarse. Por último se ha desarrollado IMS Learning Design (IMS- LD) [13] que incluye las anteriores e incorpora las facilidades para la modelación del aprendizaje a partir de las especificaciones Educational Modelling Language (EML) [14] desarrollada en la Open University of the Holanda.

IMS LD permite modelar estructuras de aprendizaje más complejas, con la participación de muchos tipos de usuarios que pueden desarrollar actividades docentes en paralelo, dependiendo de condiciones y con la posibilidad intercambiar información y mensajería. Estas son las especificaciones mas completas que existen en la actualidad para garantizar la estandarización y reusabilidad de las estructuras de aprendizaje, pero poseen un alto nivel de complejidad tanto para su uso por parte de los profesores como para su implementación mediante herramientas de software. Existen varias proyectos de investigación e instituciones que trabajan y desarrollan aplicaciones que implementan estas especificaciones en sus diferentes niveles [15], sin embargo la mayoría se concentra en la implementación de las especificaciones y no en el desarrollo de interfaces en las que el profesor no necesite conocer profundamente las mismas, es por ello que en nuestro trabajo proponemos un modelo que permita tanto a diseñadores como a usuarios desarrollar unidades de aprendizaje que permitan aprovechar las ventajas de los diagramas conceptuales en la modelación y gestión del conocimiento y las bondades de IMS LD para lograr unidades de aprendizaje mas complejas y reutilizables.

Antes de definir nuestro modelo hacemos una breve referencia a los conceptos generales sobre los diagramas conceptuales y a los elementos principales de las especificaciones IMS LD .

\subsection{Mapas Conceptuales (MC)}

Los elementos básicos de un diagrama conceptual son los conceptos, las palabras enlace y las proposiciones. Los conceptos son también llamados nodos que hacen referencia a cualquier cosa que puede provocarse o que existe. Según Novak [16], los conceptos son las imágenes mentales que provocan en nosotros las palabras o signos con que expresamos las regularidades. Las palabras enlace unen los conceptos y señalan los tipos de relación existente entre ambos. La proposición es la unidad semántica que une los conceptos.

En el mapa conceptual se organizan los elementos gráficamente formando cadenas semánticas y el conocimiento esta organizado lineal y jerárquicamente formando agrupaciones holísticas de forma que tal cuando se activa una se activa el resto. (Fig 1). 


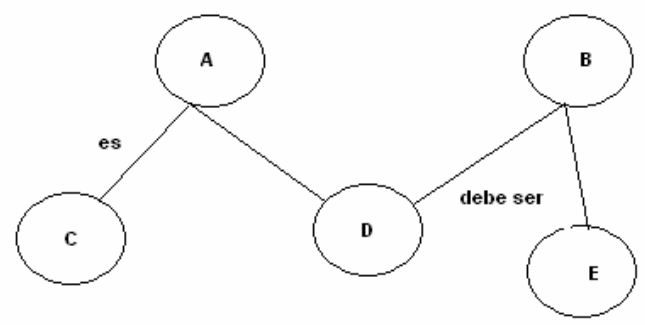

Fig. 1 Representación Gráfica de un Diagrama Conceptual

Los mapas conceptuales pueden ser descritos en forma abstracta donde los nodos ligados por arcos pueden ser vistos como hipergrafos ordenados. Pueden ser también representados mediante diagramas o por medio de lenguajes visuales [2].

Por la propia naturaleza de los $\mathrm{MC}$, las estructuras jerárquicas son las mas usadas para representarlos, pero pueden definirse otras estructuras en forma de araña, secuenciales, o en forma de sistema donde se adicionan entradas y salidas que alimentan cada uno de los conceptos del mapa. También existen los MC hipermediales que contienen una serie de conceptos relacionados entre si por medio de palabras enlace [17]. Existe gran cantidad de Software que permite a profesores y estudiantes diseñar mapas conceptuales . Muchas de estas son de libre uso y ofrecen al usuario muchas facilidades para el diseño y utilización por lo que constituye una técnica muy difundida y empleada en la gestión el conocimiento. A pesar de sus ventajas ya experimentadas por muchos, estos poseen limitaciones explicaremos mas adelante.

\subsection{Estandarización de las estructuras de aprendizaje}

Como ya explicamos existen varias especificaciones que permiten modelar y describir una estructura de contenidos, con el fin de garantizar que estas sean portables y reutilizables en los diferentes entornos educativos. A continuación hacemos una breve referencia a los elementos principales que componen el "IMS Learning Design" (IMLD- LD).

IMS-LD permite describir la estructura de tareas y actividades que ocurren en una unidad de aprendizaje. Como en todas los especificaciones, se utiliza el formato XML para describirla. Existe una documentación rigurosa y precisa [13] sobre cada unos de los componentes de esta especificación por lo que no es necesario detenernos en ello.

Una unidad de Aprendizaje basada en IMS- LD esta compuesta por objetivos, roles (pueden ser estudiantes o profesores), actividades de aprendizaje organizadas mediante diferentes estructuras que están a su vez formadas por objetos de aprendizaje y servicios [18]. (Fig 2 )

En IMS-LD se necesita conocer como los diferentes roles de usuarios realizan las actividades organizadas en estructuras coherentes [18]. (Fig. 3 )

Estas especificaciones poseen tres niveles de implementación, el nivel A, lo hemos explicado anteriormente y es el que define el vocabulario y los metadatos necesarios para la especificación. El nivel B permite asociar propiedades y condiciones para la 
ejecución de las actividades y el nivel C para implementar notificaciones y mensajes entres los componentes de la unidad de aprendizaje.

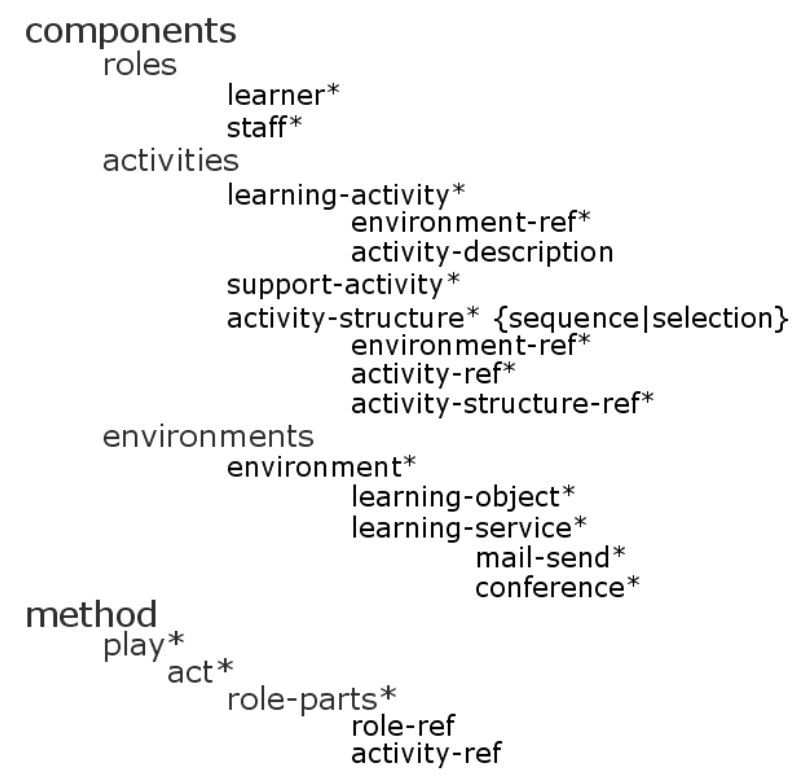

Fig. 2 Componentes de la especificación IMS-LD

Lo óptimo sería que un profesor pudiera escribir una unidad de aprendizaje utilizando los esquemas definidos en las especificaciones, pero es imposible dada la complejidad conceptual de las mismas y por los niveles de abstracción que son necesarios, es por ello que han surgido herramientas y proyectos que intentan ayudar a diseñar unidades de aprendizaje utilizando las especificaciones como base. La mayoría del software existente se encuentra en fase de validación y posee interfaces inadecuadas, muy complejas ligadas a las estructuras XML que poseen las especificaciones, lo que provoca el rechazo de los usuarios. También se desarrollan bibliotecas de funciones y componentes que permiten a los desarrolladores, crear facilidades para el uso de estas especificaciones en las aplicaciones que estos desarrollan [15].

\section{Mapas conceptuales interoperables}

Como hemos explicado, las investigaciones demuestran, la efectividad de los mapas conceptuales en el perfeccionamiento del aprendizaje, así como la experiencia adquirida por profesores y alumnos en el manejo de estas técnicas, sin embargo es importante destacar que durante las etapas de diseño el profesor necesita asociar los conceptos de un mapa con información concreta que garantice que el MC no pierda el sentido, lo que resulta realmente difícil tanto en las bibliotecas personales como In- 
ternet sobre todo cuando la información docente no está correctamente clasificada. Cuando el mapa es exportado en forma de sitio web para que sea visible en los navegadores, se pierde de cierta forma la estructura de los mapas y la posibilidad de reutilizarlos completamente o una parte de ellos para generar un nueva unidad de aprendizaje. Es por ello que nosotros proponemos un modelo que permita a profesores y diseñadores combinar el diseño de un mapa conceptual con las facilidades que ofrecen los objetos de aprendizaje, con el fin de garantizar la búsqueda y portabilidad de los mismos.

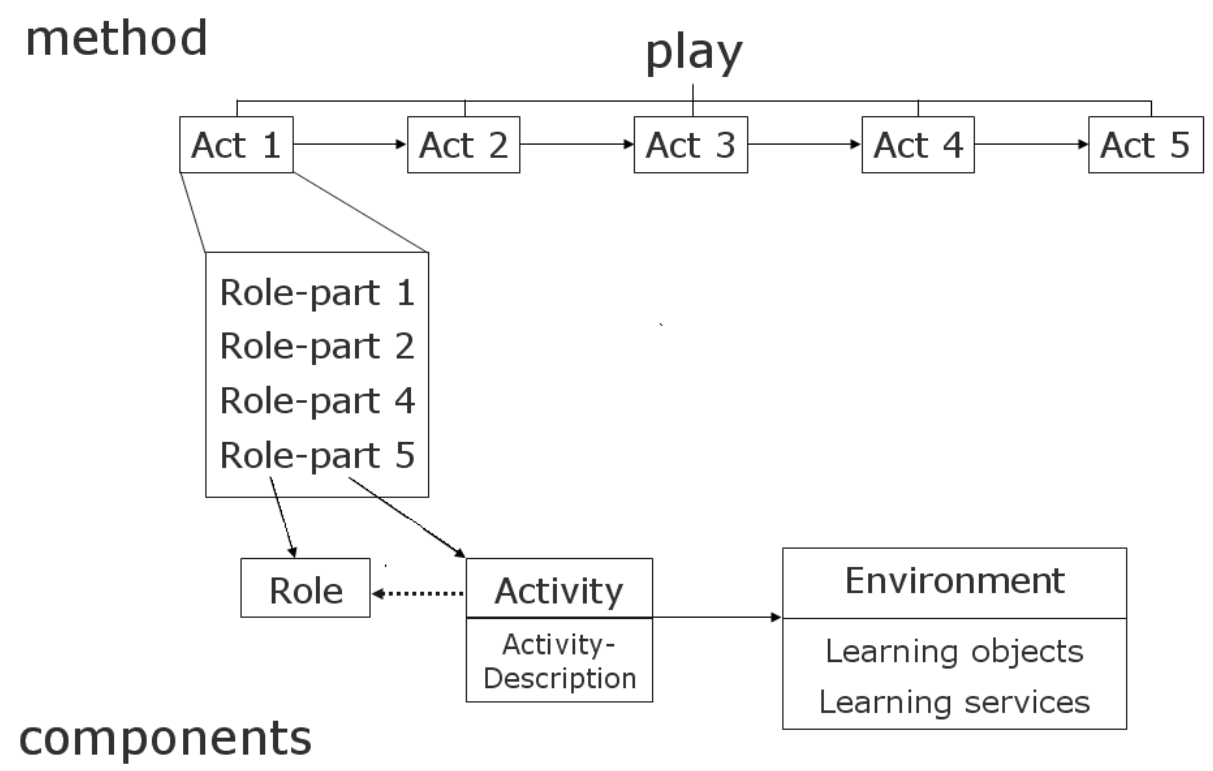

Fig 3. Esquema de Funcionamiento de IMS-LD

Nuestro modelo se basa en la posibilidad de aplicar una trasformación $\mathrm{T}$ a un mapa conceptual tradicional para convertirlo en un mapa conceptual mucho mas potente, compatible en diferentes entornos y enriquecido con las facilidades que brindan las especificaciones. Se supone que el usuario dispone de uno o varios mapas conceptuales creados con cualquiera de las herramientas disponibles para ello y que tiene acceso a repositorios de objetos de aprendizaje (LO), de modo que se pueda establecer una correspondencia entre cada nodo o concepto del MC con un objeto de aprendizaje. Cada enlace es considerado como una condición que cumpla las especificaciones IMS-LD (Fig. 4) 


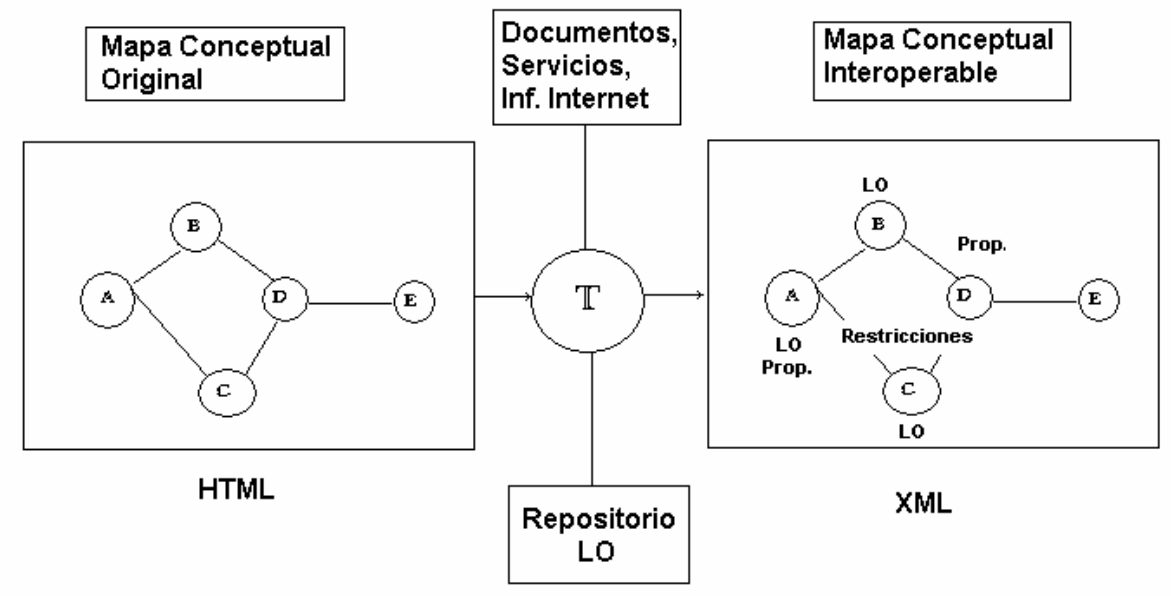

Fig 4. Relación entre un Mapa Conceptual y las especificaciones IMS-LD

Como se puede observar en la figura, durante la transformación un concepto puede asociarse a documentos en diferentes formatos que estén al alcance del diseñador o a información de internet como se hace en la forma tradicional y también a un repositorio de Objetos de aprendizaje que le permita gestionar de forma más eficiente, la información para ese concepto. El objeto de aprendizaje (LO) puede ser simple o una estructura de aprendizaje compatible con las especificaciones de diseño que hemos descrito .

Existen procedimientos y algoritmos de búsqueda muy eficientes para las estructuras XML que definen un LO y además se pueden buscar alternativas para lograr ciertos niveles de inteligencia en los gestores de LO de modo que estos puedan crear objetos de aprendizaje nuevos a partir de la información existente en el repositorio. Nosotros trabajamos en la creación de un conjunto de herramientas y servicios que automatizan la gestión de un Repositorio de LO y desarrollaremos investigaciones que nos permitan aplicar los conceptos de Ontologías en la gestión del conocimiento tomando como base la teoría sobre Objetos de aprendizaje, lo cual podría ser muy útil en este proceso.

Los nodos pueden hacer referencias a servicios como CHAT, Email o solicitar una determinada tarea de cómputo más compleja. A cada uno de los nodos se le pueden asignar propiedades (prop.) que caractericen el nodo o concepto y definir indicadores que puedan ser utilizados como condiciones o restricciones en la transición de un nodo a otro.

Se pueden diseñar aplicaciones que se basen en el modelo anterior, cuya entrada sea el mapa conceptual y la salida seria el mapa conceptual enriquecido con bondades de las especificaciones IMS-LD, lo cual garantiza su portabilidad y mayor potencia como hemos planteado.

Hemos creado una aplicación que llamamos COMALO (from COncept MAps to Learning Objects) que genera estructuras de aprendizaje compatibles con las especi- 
ficaciones de diseño IMS- CP y IMS-LD en los niveles A y B. Esta aplicación utiliza un repositorio de objetos de aprendizaje y una estructura XML que representa el mapa conceptual que introduce el usuario mediante una interfaz mas cercana a sus conocimientos, sin abligarlo a conocer en detalle los términos utilizados en la especificaciones.

COMALO permite buscar en el repositorio y asociar los Objetos de Aprendizaje a los nodos, se pueden definir propiedades y transiciones que pueden estar sujetas a restricciones.

A pesar de que la aplicación esta en fase de desarrollo es posible utilizarla en la generación de mapas conceptuales que cumplan con las especificaciones IMS LD en los niveles A y B.

\section{Conclusiones y Recomendaciones}

En nuestro trabajo hemos propuesto una alternativa que puede servir de base a desarrolladores de software y pedagogos, en el diseño de aplicaciones orientadas a la gestión del conocimiento porque existen las condiciones necesarias para poder implementar y llevar a la práctica las recomendaciones que se hemos planteado con el fin de producir mapas conceptuales reutilizables bajo los diferentes entornos educativos .

La vinculación de ambas teorías permitirá a los usuarios elegir información más precisa, clasificada y estructurada, acorde a la complejidad de los procesos de aprendizaje de nuestros tiempos.

Como hemos explicado, las herramientas actuales que implementan la especificaciones de diseño y empaquetamiento de contenidos, son todavía muy orientadas a los usuarios que las dominan, lo cual impide su desarrollo e implica rechazo por parte del resto de usuarios, incluso de los mas avanzados. El uso de mapas conceptuales como base para el diseño permitirá sin dudas, lograr mejores resultados .

La efectividad de la herramienta COMALO será validada mediante el diseño de cursos de español a partir del repositorio de objetos de aprendizaje que posee el Aula Virtual de Español ( AVE).

Es necesario trabajar en la interfaz grafica de esta aplicación para ofrecer mejores facilidades de diseño y trabajar intensamente en el desarrollo de interpretes que permitan utilizar las estructuras IMS-LD generadas, sobre todo en los niveles B y C donde hay muy pocos resultados.

\section{References}

1. Uñantes G. , E-learning: cambiando paradigmas en capacitación. 2003, El principe.com. $\mathrm{http}: / /$ www.elprincipe.com/teleformacion/notas/index 14.shtml

2. Estrada , V., Mapas Conceptuales. 1998, MES.

3. Morales, R., Capacitación basada en objetos reusables de aprendizaje. 2001. http://www.umb.edu.co/umb/sitiopedagogia/lecturas/tendencias.pdf 
4. IMS Global Learning Consortium, IMS Learning Resource Meta-Data Specification: Version 1.1 Final Specification. 2000, IMS Global Learning Consortium. http://www.imsproject.org/metadata/index.html

5. ADL, Sharable Content Object Reference Model Version 1.2. 2001, Advanced Distributed Learning. http://www.adlnet.org

6. LTSC, Draft Standard for Learning Object Metadata Version 6.1. 2001, Learning Technology Standards Committee. http://ltsc.ieee.org/doc

7. Willey, D., Connecting learning objects to instructional design theory: A definition, a metaphor, and a taxonomy,. 2001. http://reusability.org/read/chapters/wiley.doc

8. Instituto Cervantes , Pagina Web del Instituto Cervantes. 2004. http://www.cervantes.es/

9. Marco, M.y.Moron, D., Sistema de producción multimedia para la WEB, ejemplo aula virtual del español. 2003: MIC, CUBA.

http:/www.informaticahabana.co.cu/eventos/eventos/Educacion/default.htm

10. Iriarte, L., Marco, M., Morón, D., Creación automatizada de una Biblioteca de Objetos de aprendizaje. 2003, Memorias del X CONGRESO INTERNACIONAL DE INFORMÁTICA EN LA EDUCACIÓN.

11. IMS Global Learning Consortium, IMS Content Packaging.Version 1.1.3 Final Specification 2003.

http://www.imsglobal.org/content/packaging/cpv1p1p3/imscp_infov1p1p3.html

12. IMS Global Learning Consortium, IMS Simple Sequencing Information and Behavior Model. Version 1.0 Final Specification. 2003.

$\mathrm{http} / / /$ www.imsglobal.org/simplesequencing/ssv1p0/imsss_infov1p0.html

13. IMS Global Learning Consortium, IMS Learning Design Best Practice and Implementation Guide.Version 1.0 Final Specification. 2003.

http://www.imsglobal.org/learningdesign/ldv1p0/imsld_bestv1p0.html

14. OpenUniversiteItNetherlands, Educational Modelling Language. 2004. http://eml.ou.nl/eml-ou-nl.htm

15. Britain, S., A Review of Learning Design: Concept, Specifications and Tools. 2004, A report for the JISC E-learning Pedagogy Programme.

http://www.jisc.ac.uk/uploaded_documents/ReviewLearningDesign.doc

16. Novak, J.D., Aprendiendo a Aprender. 1988: Ediciones Martinez Roca, S.A.

17. Gaines, B.e.S., Collaboration Through Conceptt Maps. 1995.

18. Olivier, B., IMS Learning Design Public Draft .An Overview. 2003, CETIS. http://www.brookes.ac.uk/research/odl/alt-nl03/Presentations/LD presentation.ppt 